\title{
Feature Generation Method by Geometrical Interpretation of Fisher Linear Discriminant Analysis
}

\author{
Tadahiro Oyama Non-member (The University of Tokushima, oyama@is.tokushima-u.ac.jp) \\ Yuji Matsumura Non-member (The University of Tokushima, yuu@is.tokushima-u.ac.jp) \\ Stephen Githinji Karungaru Non-member (The University of Tokushima, karunga@is.tokushima-u.ac.jp) \\ Minoru Fukumi Member (The University of Tokushima, fukumi@is.tokushima-u.ac.jp)
}

Keywords: simple-FLDA, simple-PCA, pattern recognition, EMG, face recognition, feature generation

In this paper, we propose an approximation algorithm (Simple-FLDA) that calculates eigenvectors of the fisher linear discriminant analysis sequentially by an easy iterative calculation without the use of the matrix calculation. In particular, the point to derive an approximation algorithm is to consider geometrical interpretation of the maximization of the variance between classes and minimization of variance in each class. The algorithm of Simple-FLDA is shown as follows.

First, the maximization of the variance between classes as a description of this algorithm is described. The mean vector $h_{j}$ of the data of each class is calculated. The following calculations are carried out by using this $h_{j}$.

$$
\begin{aligned}
& y_{n}=\left(a_{n}^{k}\right)^{T} h_{j} \ldots \ldots \ldots \ldots \ldots \ldots \ldots \ldots \ldots \ldots \ldots \ldots \ldots \\
& f\left(y_{n}, h_{j}\right)=\left\{\begin{array}{ll}
h_{j} & \text { if } y_{n} \geq 0 \\
-h_{j} & \text { otherwise }
\end{array} \ldots \ldots \ldots \ldots \ldots \ldots \ldots\right.
\end{aligned}
$$

where $a_{n}^{k}$ is an approximated vector of the $n$-th eigenvector, and $k$ shows an index of repetition of the calculation. The threshold function(2) is summed using every mean vector. It can converge to the eigenvector that maximizes the variance between classes by these formulas. Eq.(2) can be replaced by another forms.

Next, we describe the algorithm of the minimization of variance in each class. The vector $x_{j}$ s have zero mean in the class. The positional relation between data $x_{j}$ and an arbitrary vector assumed to be $a_{n}^{k}$ is considered. The direction where $x_{j}$ is projected and vector length is minimized is the orthogonal direction to $x_{j}$. Therefore, direction $b_{j}$ where the direction component of $x_{j}$ was removed from $a_{n}^{k}$ can be expressed by the following eq.(3).

$$
\begin{aligned}
& b_{j}=a_{n}^{k}-\left(\hat{x}_{j} \cdot a_{n}^{k}\right) \hat{x}_{j} \ldots \ldots \ldots \ldots \ldots \ldots \ldots \ldots \ldots \\
& \hat{x}_{j}=\frac{x_{j}}{\left\|x_{j}\right\|}
\end{aligned}
$$

This is the same as schmidt orthogonalization procedure. An actual quantity is obtained by normalization of the vector length of $b_{j}$ as follows.

$$
f_{i}\left(b_{j}, x_{j}\right)=\frac{\left\|x_{j}\right\|}{\left\|b_{j}\right\|} b_{j}
$$

The averaging is carried out by using all input vectors in each class during the repetition calculation. In other words, the influence of a component that the norm of a vector is
Table 1. Recognition accuracy in the personal authentication without expression

\begin{tabular}{|c|c|c|c|c|c|}
\hline No.of subjects & 10 & 20 & 30 & 40 & 50 \\
\hline S-PCA & 96.7 & 97.5 & 98.3 & 98.3 & 98.7 \\
\hline S-FLDA & 98.3 & 100.0 & 100.0 & 99.2 & 99.7 \\
\hline
\end{tabular}

large in this eq.(5) is significant. Therefore, it is hoped that it converges to a direction to minimize the variance in each class. The repetition calculation is shown by the following formulas.

$$
\begin{aligned}
& f_{n}^{k}=\sum_{i=1}^{c} N_{i} f\left(y_{n}, h_{i}\right)+\sum_{i=1}^{c} \sum_{j=1}^{N_{i}} f_{i}\left(b_{j}, x_{j}\right) \ldots \ldots \ldots \ldots \ldots \ldots \ldots \ldots \ldots \ldots \ldots \ldots \ldots \ldots \ldots \ldots \ldots \ldots \ldots
\end{aligned}
$$

where $c$ is the number of classes and $N_{i}$ shows the number of data in class $i . N_{i}$ in the first term is used for equalizing the number of data in both terms. Thus, an arbitrary vector is converged to the eigenvector by achieving maximization of the variance between classes and minimization of the variance in each class at the same time.

We carry out computer simulations on recognition of wrist motion patterns by EMG measured from wrist and personal authentications that use face images to verify the effectiveness of this technique. For instance, result in the personal authentication is shown in Table 1.

As a result, recognition accuracy by the Simple-FLDA is better than that by the Simple-PCA in each experiment. It was confirmed that the Simple-FLDA was one of the effective techniques in the pattern recognition. We will confirm the effectiveness even for another problems in the future. Furthermore convergence property of the Simple-FLDA should be analyzed. 


\section{Feature Generation Method by Geometrical Interpretation of Fisher Linear Discriminant Analysis}

$\begin{array}{ll}\text { Tadahiro Oyama* } & \text { Non-member } \\ \text { Yuji Matsumura* } & \text { Non-member } \\ \text { Stephen Githinji Karungaru* } & \text { Non-member } \\ \text { Minoru Fukumi* } & \text { Member }\end{array}$

This paper presents a new algorithm for feature generation, which is derived based on geometrical interpretation of the fisher linear discriminant analysis (FLDA). This algorithm (Simple-FLDA) is an approximation algorithm that calculates eigenvectors sequentially by an easy iterative calculation by expressing the maximization of variance between classes and minimization of variance in each class without the use of matrix calculation. We carry out computer simulations about recognition of wrist motion patterns by EMG measured from wrist and personal authentications that use face images to verify the effectiveness of this technique. The result was compared with the result of principal component analysis (Simple-PCA).

Keywords: simple-FLDA, simple-PCA, pattern recognition, EMG, face recognition, feature generation

\section{Introduction}

In the past, the principal component analysis(PCA), the fisher linear discriminant analysis(FLDA), and the factor analysis ${ }^{(1)}$ have been widely used as statistical techniques in pattern recognition. However, in recent years, many new statistical techniques in the pattern recognition have been proposed. For instance, techniques based on a kernel function were proposed $^{(2)(3)}$. In particular, those effectiveness is shown in the field such as reduction of influence of light and recognition of face. Belhumeur et al. ${ }^{(4)}$ showed the effectiveness of the Fisherface by comparing the Eigenface and the Fisherface. However, a lot of zero eigenvalues exist because the size of an image is large when the Fisherface is derived. Therefore, the Fisherface cannot be derived directly. Thus, after its data dimension is compressed by PCA, the eigenvector of the Fisherface is calculated. As a result, enormous calculation amount to execute matrix calculation is needed. In addition, PCA is a linear transformation that maximizes the mutual information. Therefore, principal components are found to maximize the variance of data. On the other hand, the discriminant analysis (fisher linear discriminant analysis) is a technique to find eigenvectors that achieve maximization of variance between classes and minimization of variance in each class at the same time. Therefore, the inversion matrix of the covariance matrix of data in each class is necessary. When an image signal is used, a lot of zero eigenvalues exist because the dimension of the image is enormously larger than the number of data. Thus, we have to compress dimension of data by PCA as a prepro-

\footnotetext{
* Department of Information \& Science Intelligent Systems, The University of Tokushima

2-1, Minami-josanjima, Tokushima, 770-8506
}

cessing because the calculation of the inversion matrix is difficult to be done. In addition, when the application to industry is considered, it is important to extract features as much as possible by an easy algorithm without using matrix calculation.

On the other hand, some simple iterative calculation techniques were proposed to reduce a huge amount of matrix calculation in PCA. In the field of neural networks (NNs), methods by Sanger ${ }^{(5)}$ and Kung ${ }^{(6)}$ are famous. Moreover, Partridge et al. ${ }^{(7)}$ have proposed easy Simple-PCA compared to them. It is a simple iterative calculation algorithm. This Simple-PCA is an approximation algorithm by which the principal component vectors can be sequentially calculated from the 1st principal component. As for this Simple-PCA, its effectiveness has been shown in many applications. In the field of face information processing, the use of it as feature extraction is more than the use as dimension compression ${ }^{(8)(9)}$. However, the distribution between classes is not considered at all because the Simple-PCA is feature generation based on the principal component vector. It is not necessarily a feature generator suitable for pattern classification.

Therefore, we paid attention to the fisher linear discriminant analysis whose feature extraction function is superior than the principal component analysis in pattern classification of the face information processing etc. In this paper, we propose an approximation algorithm (Simple-FLDA) that calculates eigenvectors sequentially by an easy iterative calculation without the use of matrix calculation in the discriminant analysis. In this proposal technique, the eigenvectors of the fisher linear discriminant analysis can be sequentially calculated by an easy repetition operation by expressing the maximization of variance between classes and minimization of variance in 
each class using separate repetition formulas. In particular, the point to derive an approximation algorithm is to consider geometrical interpretation of the maximization of the variance between classes and the minimization of the variance in each class. We carry out computer simulations on recognition of wrist motion patterns by EMG measured from wrist and personal authentications that use face images to verify the effectiveness of this technique. The results are compared with the result by the principal component analysis (Simple-PCA).

\section{Simple-FLDA}

2.1 Simple-PCA The Simple-PCA (Simple principal component analysis) is a technique which is proposed by Partridge and others ${ }^{(7)}$ to speed up the principal component analysis. The technique was an approximation algorithm by which the principal components can be sequentially found from the first component. Moreover, its effectiveness is confirmed in many fields such as recognition of hand-written character $^{(7)}$ and dimensionality reduction of models for information retrieval $^{(10)}$.

The algorithm of this technique sequentially solves for eigenvectors that maximizes the variance over all samples. Concretely, it is summarized as the following. First of all, a set of input vectors is defined as follows.

$$
v=\left\{v_{1}, v_{2}, \cdots, v_{m}\right\}
$$

To make the center of gravity of this set the origin, the calculation shown in eq.(3) is performed, and a new set of vectors (2) are obtained.

$$
\begin{aligned}
X & =\left\{x_{1}, x_{2}, \cdots, x_{m}\right\} \\
x_{i} & =v_{i}-\frac{1}{m} \sum_{j=1}^{m} v_{j} \cdots
\end{aligned}
$$

Next, the following output function is used to yield an output.

$$
y_{n}=\left(\alpha_{n}\right)^{T} x_{j}
$$

where $\alpha_{n}$ is the eigenvector that shows the $n$-th principal component. If the input vector $x_{j}$ is the same direction as $\alpha_{n}$, the function shown by eq.(4) outputs a positive value. However, if it is the opposite direction, a negative value is outputted. Thus, the following threshold function is introduced.

$$
f\left(y_{n}, x_{j}\right)= \begin{cases}x_{j} & \text { if } y_{n} \geq 0 \\ -x_{j} & \text { otherwise }\end{cases}
$$

The initial vector $a_{n}^{1}$ initialized by arbitrary random values is brought close in the same direction as $\alpha_{n}$ by these functions and the repetition operation shown in the eq.(6)(7).

$$
\begin{aligned}
& a_{n}^{k+1}=\frac{\sum_{j} f\left(y_{n}, x_{j}\right)}{\left\|\sum_{j} f\left(y_{n}, x_{j}\right)\right\|} \\
& y_{n}=\left(a_{n}^{k}\right)^{T} x_{j} \ldots \ldots \ldots
\end{aligned}
$$

where $k$ is an index of repetitions and $a_{n}^{k+1}$ is a vector after $k+1$ times iteration. The value of the output function is calculated by using $a_{n}^{k}$ that is the previous calculation result. This repetition calculation is continued until $a_{n}^{k+1}$ is converged. This vector after convergence is an eigenvector (approximated eigenvector).

When the next eigenvector is calculated, it is necessary to calculate it by using new vectors $x_{j}$ 's after the previous principal component is removed from the input vectors by doing the calculation shown in eq.(8).

$$
x_{j}^{\prime}=x_{j}-\left(a_{n}^{k+1} \cdot x_{j}\right) a_{n}^{k+1}
$$

After the component is removed, the principal component can be evaluated by repeating a similar calculation in order with a high contribution relevance.

2.2 Simple-FLDA The fisher linear discriminant analysis is one of techniques of discriminant analysis. This technique can find eigenvectors that achieve maximization of variance between classes and minimization of variance in each class at the same time. However, matrix calculation cost becomes huge and a calculation time becomes very long. Simple-FLDA (Simple Fisher Linear Discriminant Analysis) is an algorithm from which eigenvectors are found without using matrix calculation, and by using the repetition calculation of an approximation algorithm.

The Simple-FLDA is an approximated algorithm that achieves maximization of the variance between classes and minimization of the variance in each class at the same time. The Simple-FLDA in this paper separately represents both the terms and is derived as an approximated algorithm based on them. First, the maximization of the variance between classes as a description of this algorithm is described. It is regard as the SimplePCA using mean vectors. The mean vector $h_{j}$ of the data of each class is calculated. In Fig. $1, h_{j}$ is shown as a black dot. The mean value of all data is assumed to be 0 . The following calculations are carried out by using this $h_{j}$.

$$
\begin{aligned}
& y_{n}=\left(a_{n}^{k}\right)^{T} h_{j} \ldots \ldots \ldots \ldots \ldots \\
& f\left(y_{n}, h_{j}\right)= \begin{cases}h_{j} & \text { if } y_{n} \geq 0 \\
-h_{j} & \text { otherwise }\end{cases}
\end{aligned}
$$

where $a_{n}^{k}$ is an approximated vector of the $n$-th eigenvector, and $k$ shows an index of repetition of the calculation. In Fig.1, a dotted line shows a line (hyperplane) which adjusts eq.(9) to 0 . The threshold function(10) is summed using every mean vector. It can converge to the eigenvector that maximizes the variance between classes by these formulas. Eq.(10) can be replaced by another forms ${ }^{(7)}$.

Next, we describe the algorithm of the minimization of variance in each class. In Fig.2, data vector $x_{j}$ in a class is shown as a black dot. The vector $x_{j}$ s have zero mean in the class. The positional relation between data $x_{j}$ and an arbitrary vector assumed to be $a_{n}^{k}$ is considered. The direction where $x_{j}$ is projected and vector length is minimized is the orthogonal direction to $x_{j}$. Therefore, direction $b_{j}$ where the direction component of $x_{j}$ 


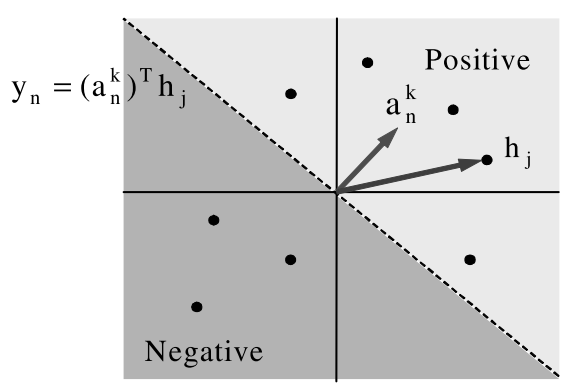

Fig. 1. Maximization of between-class variance

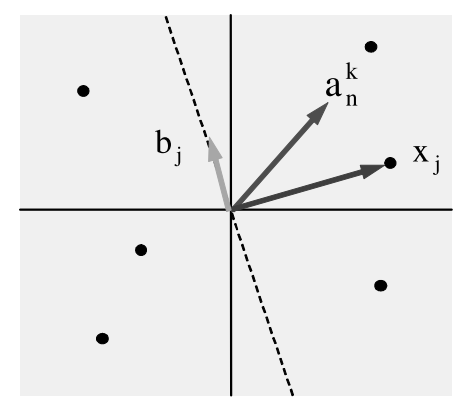

Fig. 2. Minimization of within-class variance

was removed from $a_{n}^{k}$ can be expressed by the following eq.(11).

$$
\begin{aligned}
& b_{j}=a_{n}^{k}-\left(\hat{x}_{j} \cdot a_{n}^{k}\right) \hat{x}_{j} \ldots \ldots \ldots \ldots \ldots \ldots \ldots \ldots \ldots \ldots \ldots \ldots \ldots \ldots \ldots \ldots \ldots \ldots \ldots \ldots \ldots
\end{aligned}
$$

This is the same as schmidt orthogonalization procedure. An actual quantity is obtained by normalization of the vector length of $b_{j}$ as follows.

$$
f_{i}\left(b_{j}, x_{j}\right)=\frac{\left\|x_{j}\right\|}{\left\|b_{j}\right\|} b_{j} \ldots \ldots \ldots \ldots \ldots \ldots \ldots
$$

In Fig. $2, b_{j}$ is the same direction as the dotted line which is perpendicular to $x_{j}$, and it can achieve exactly zero for the variance of $x_{j}$. The averaging is carried out by using all input vectors in each class during the repetition calculation. In other words, the influence of a component that the norm of a vector is large in this eq.(13) is significant. Therefore, it is hoped that it converges to a direction to minimize the variance in each class. The repetition calculation is shown by the following formulas.

$$
\begin{aligned}
& f_{n}^{k}=\sum_{i=1}^{c} N_{i} f\left(y_{n}, h_{i}\right)+\sum_{i=1}^{c} \sum_{j=1}^{N_{i}} f_{i}\left(b_{j}, x_{j}\right) \cdots \cdots \\
& a_{n}^{k+1}=\frac{f_{n}^{k}}{\left\|f_{n}^{k}\right\|} \ldots \ldots \ldots \ldots \ldots \ldots \ldots \ldots \ldots \ldots \ldots
\end{aligned}
$$

where $c$ is the number of classes and $N_{i}$ shows the number of data in class $i . \quad N_{i}$ in the first term is used for equalizing the number of data in both terms. Thus, an arbitrary vector is converged to the eigenvector by achieving maximization of the variance between classes and minimization of the variance in each class at the same time.

When the next eigenvector is calculated after it converges, it is necessary to remove the component of the previous eigenvector from the input vectors as well as the Simple-PCA. Moreover, it is necessary to remove the component from the initial vector of the next eigenvector and the mean vector of each class. In addition, $k$ changes considerably depending on the convergence condition.

\section{Computer Simulation}

3.1 Wrist Motion Recognition Experiment Using EMG In this section, recognition experiments of wrist motions are conducted by using EMG measured from the wrist to verify the effectiveness of the Simple-FLDA. First, the system configuration for experiment is explained. Next, the result of computer simulations is described.

3.1.1 EMG Recognition System The construction of an EMG pattern recognition system in this paper is shown in Fig.3. The system consists of an input part, a signal processing part, a feature extraction part and a learning discrimination part.

In the input part, EMG is measured form the wrist by electrodes in four poles. EMG in a specific frequency band region is extracted through a lowpass filter in the signal processing part. In addition, after these extracted signals are amplified with the amplifier, they are converted to digital signals by the A/D conversion. Next, the signals are transformed into the power spectra in frequency components of EMG by the FFT processing in the feature extraction part. Finally, the transformed signals are discriminated by the discrimination circuit such as a neural network in the learning discrimination part. They are explained in detail as follows.

(a) Input part

In this paper, we use the surface electrode in consideration of practical application. Furthermore, because its treatment is easy, a dry-type electrode is adopted though surface electrodes are divided into the wet-type (used electrolysis cream) and dry-type (non-used electrolysis cream). Moreover, the silver chloride is suitable for the material of the dry-type electrode because of a low electrode impedance in low frequency range. However, the copper plate coated with rolled gold was used because we couldn't obtain the silver chloride. The electrode is attached from the aspect of convenience in the wrist though it is necessary to place in flexor carpi radialis muscle and flexor carpi ulnaris muscle that can strongly measure EMG in high accuracy. The motions of the wrist at this time are set in seven states of the neutral, up, down, right, left, inside and outside as shown in Fig.4 and measured in each state.

(b) Signal processing part

In general, it was reported that from a few $[\mathrm{Hz}]$ to $2[\mathrm{kHz}]$ frequency of the EMG were important ${ }^{(11)}$. Then, the frequency components of $70[\mathrm{~Hz}]$ or more with low possibility that the noise mixes is detected with the lowpass filter and the notch filter to avoid the influence of the commercial frequency noise $(60[\mathrm{~Hz}])$. Thereby, the 


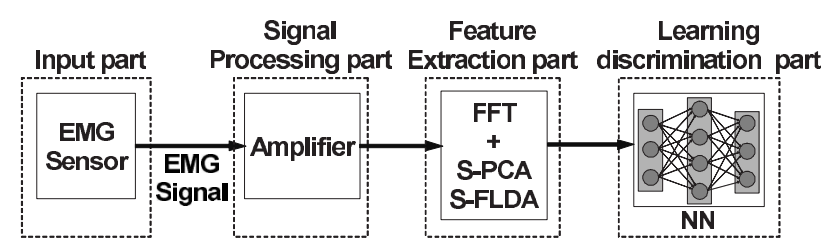

Fig. 3. Structure of the EMG recognition system

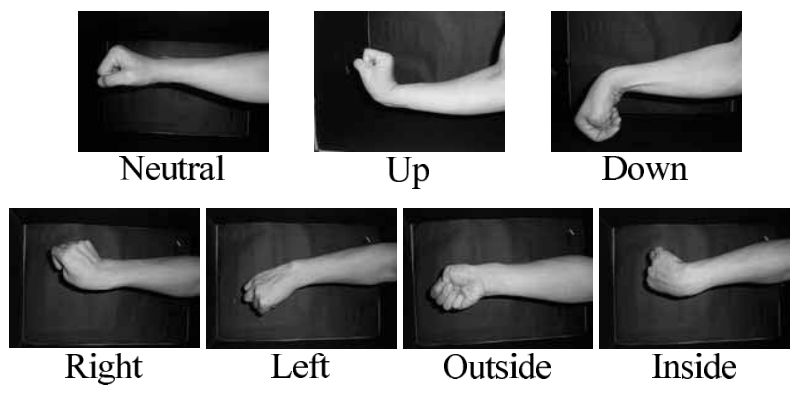

Fig. 4. Motion patterns of wrist

signal between $70[\mathrm{~Hz}]$ and $2[\mathrm{kHz}]$ is extracted from measured EMG. In addition, this extracted signal is amplified with a differential amplifier.

(c) Feature extraction part

The signal taken in the signal processing part is translated into the power spectra in the frequency component of EMG by performing FFT. This is 1024 points FFT. It is executed ten times moving a data window every 512 data point. The mean value is used as data when the next processing is executed. Moreover, the following learning discrimination part uses the frequency component of $1[\mathrm{kHz}]$ or less from the earlier study ${ }^{(12)}$.

(d) Learning discrimination part

In the learning discrimination part, wrist motions are discriminated based on the signal from the feature extraction part. A neural network $(\mathrm{NN})$ is used as a method of classifying these signals because nonlinearity is included in EMG. NN used is composed of three layers (input layer, hidden layer and output layer). Moreover, the back-propagation algorithm is used for learning.

3.1.2 Experimental Conditions In this section, we experimented the effectiveness of the present method for classifying EMG signals. Data obtained by the FFT processing to the EMG signals is used for the input. In recognition, it is decided that it is a correct answer when a corresponding neuron in the output layer corresponded to an input motion pattern has fired most greatly. The number of input layer units is 412 because we use a part where change of frequency component of $1[\mathrm{kHz}]$ or less than it is violent from the earlier study ${ }^{(12)}$. Moreover, the number of hidden layer units and the learning factors were decided based on the result of the trial and error.

Next, we reduced the number of input data by using the Simple-PCA and the Simple-FLDA. In the SimplePCA, the number of eigenvectors become 20 when the accumulated contribution relevance is $95[\%]$ or more. Thus, we found the eigenvectors until the 20th component. The experiment was carried out by using the vectors in which the input dimension to NN was reduced to the input data to NN. Moreover, in the discriminant analysis, the number of effective eigenvectors is up to $c-1$ when the number of classes is $c$. Therefore, in the Simple-FLDA, we found eigenvectors until the 6 th as input data of NN because the number of classes is 7 . At the same time, the recognition experiment that used the technique of the minimum distance classifier without using $\mathrm{NN}$ was carried out. In this experiment, three subjects A (male: 30 years old), B (male: 22 years old), C (male: 21 years old) data were used.

3.1.3 Experimental Result Table 1 shows the recognition results. The experimental results to subjects $\mathrm{A}, \mathrm{B}$ and $\mathrm{C}$ are shown in (a), (b) and (c), respectively. In tables, S-PCA and S-FLDA are results obtained by the minimum distance classifier. S-PCA \& NN and SFLDA \& NN are results obtained by NN. As a result, compared to NN, it is found that total recognition accuracy was improved by using the Simple-PCA and the Simple-FLDA. Therefore, it is thought that the SimplePCA and the Simple-FLDA are effective techniques in the EMG recognition. Moreover, although there is a variation in data, the Simple-FLDA is overall higher than the Simple-PCA in recognition accuracy. When these techniques are compared, it can be said that the Simple-FLDA is superior in recognition of the EMG.

3.2 Face Recognition Experiment In this section, face recognition experiment was carried out to verify the effectiveness of the Simple-FLDA. Each case of personal authentications was compared with the result of the Simple-PCA.

3.2.1 Personal Authentication with Facial Expression Samples of face images used in the first personal authentication with facial expression are shown in Fig.5. Five kinds of facial expressions are neutral, laugh, surprise, anger and sadness. We tried face recognition insensitive facial expression. Each subject has two sets of five expressions. As subjects are six people, the total number of image data is 60 . The eigenvector is found by using a part of these 60 as a learning sample. The total number of learning samples is 18 as we used three images in each class (person). The evaluation was carried out by using 42 images of the remainder. The size of image data is $100 \times 100$ pixels, which are transformed into gray-scale ones with 256 levels. The image data is further compressed into 2,500 pixels. The length of eigenvectors to be obtained is therefore 2,500.

Table 2 shows the result of the recognition experiment. The number of eigenvectors becomes 10 when the accumulated contribution relevance of the Simple-PCA is 83[\%]. Recognition was done by using the minimum distance classifier. The results labeled "S-FLDA" and "SPCA" are ones obtained by the Simple-FLDA and the Simple-PCA, respectively. As shown in Table 2, recognition accuracy by the Simple-FLDA is better than that by the Simple-PCA.

In the fisher linear discriminant analysis the number of effective eigenvectors is at most $c-1$ where $c$ is the number of classes. However, the Simple-FLDA is the approximated algorithm and can have some approximation error. Therefore, we used more eigenvectors than $c-1$, 
Table 1. Recognition accuracy in the wrist motion recognition experiment

(a) Subject A (male : 30 years old)

\begin{tabular}{|c|c|c|c|c|c|}
\hline \multirow{2}{*}{ Motion } & \multicolumn{5}{|c|}{ Recognition accuracy[\%] } \\
\cline { 2 - 6 } & NN & S-PCA & $\begin{array}{c}\text { S-PCA } \\
\text { \&NN }\end{array}$ & S-FLDA & $\begin{array}{c}\text { S-FLDA } \\
\text { \&NN }\end{array}$ \\
\hline Neutral & 96.7 & 90.0 & 93.3 & 96.7 & 95.0 \\
\hline Up & 68.3 & 93.3 & 93.3 & 91.7 & 95.0 \\
\hline Down & 93.3 & 96.7 & 91.7 & 96.7 & 93.3 \\
\hline Right & 76.7 & 78.3 & 90.0 & 76.7 & 93.3 \\
\hline Left & 76.7 & 78.3 & 88.3 & 81.7 & 88.3 \\
\hline Inside & 61.7 & 95.0 & 96.7 & 95.0 & 95.0 \\
\hline Outside & 51.7 & 81.7 & 76.7 & 86.7 & 75.0 \\
\hline Total & 75.0 & 87.6 & 90.0 & 89.3 & 90.7 \\
\hline
\end{tabular}

(b) Subject B (male : 22 years old)

\begin{tabular}{|c|c|c|c|c|c|}
\hline \multirow{2}{*}{ Motion } & \multicolumn{5}{|c|}{ Recognition accuracy[\%] } \\
\cline { 2 - 6 } & NN & S-PCA & $\begin{array}{c}\text { S-PCA } \\
\text { \&NN }\end{array}$ & S-FLDA & $\begin{array}{c}\text { S-FLDA } \\
\& N N\end{array}$ \\
\hline Neutral & 90.0 & 93.3 & 90.0 & 88.3 & 98.3 \\
\hline Up & 58.3 & 68.3 & 66.7 & 63.3 & 66.7 \\
\hline Down & 71.7 & 83.3 & 86.7 & 90.0 & 95.0 \\
\hline Right & 73.3 & 96.7 & 96.7 & 100.0 & 100.0 \\
\hline Left & 91.7 & 93.3 & 100.0 & 96.7 & 100.0 \\
\hline Inside & 75.0 & 68.3 & 66.7 & 68.3 & 66.7 \\
\hline Outside & 90.0 & 85.0 & 83.3 & 88.3 & 81.7 \\
\hline Total & 78.6 & 84.0 & 84.3 & 85.0 & 86.9 \\
\hline
\end{tabular}

(c) Subject C (male : 21 years old)

\begin{tabular}{|c|c|c|c|c|c|}
\hline \multirow{2}{*}{ Motion } & \multicolumn{5}{|c|}{ Recognition accuracy[\%] } \\
\cline { 2 - 6 } & NN & S-PCA & $\begin{array}{c}\text { S-PCA } \\
\text { \&NN }\end{array}$ & S-FLDA & $\begin{array}{c}\text { S-FLDA } \\
\& N N\end{array}$ \\
\hline Neutral & 50.0 & 65.0 & 80.0 & 71.7 & 76.7 \\
\hline Up & 66.7 & 75.0 & 91.7 & 81.7 & 88.3 \\
\hline Down & 96.7 & 85.0 & 91.7 & 83.3 & 93.3 \\
\hline Right & 90.0 & 81.7 & 93.3 & 90.0 & 93.3 \\
\hline Left & 98.3 & 95.0 & 98.3 & 96.7 & 98.3 \\
\hline Inside & 48.3 & 83.3 & 91.7 & 85.0 & 96.7 \\
\hline Outside & 55.0 & 80.0 & 73.3 & 73.3 & 86.7 \\
\hline Total & 72.1 & 80.7 & 88.6 & 83.1 & 90.5 \\
\hline
\end{tabular}

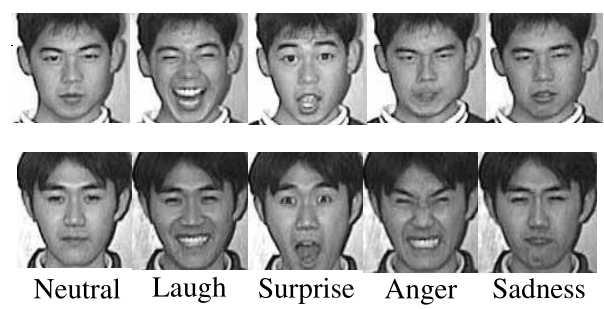

Fig. 5. Face images with expressions

Table 2. Recognition accuracy in the personal authentication with facial expression [\%]

\begin{tabular}{|c|c|c|c|c|c|c|}
\hline EVs & 1 & 2 & 3 & 4 & 5 & 10 \\
\hline S-PCA & 47.6 & 42.9 & 49.5 & 50.4 & 57.3 & 55.9 \\
\hline S-FLDA & 52.4 & 60.7 & 62.9 & 60.5 & 63.9 & 60.3 \\
\hline
\end{tabular}

and furthermore for comparison to the Simple-PCA.

3.2.2 Personal Authentication without Expression Examples of face images used in the second personal authentication experiment is shown in Fig.6. They include the database of University of Oulu. Each person has 6 images, which include faces with slight expression. The experiment is conducted by using the image of 50 persons. The size of images is $100 \times 100$ pixels. The dimension of eigenvectors becomes 2,500 after reduction to $50 \times 50$.

The number of learning data to find eigenvectors is 5 for each person. We used the leave-one-out crossvalidation method as an evaluation method. The accu-

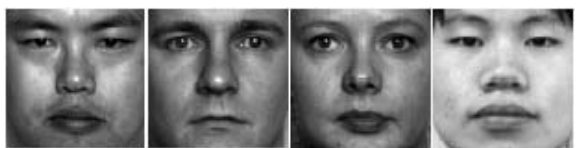

Fig. 6. Face images

Table 3. Recognition accuracy in the personal authentication without expression [\%]

\begin{tabular}{|c|c|c|c|c|c|}
\hline No.of subjects & 10 & 20 & 30 & 40 & 50 \\
\hline S-PCA & 96.7 & 97.5 & 98.3 & 98.3 & 98.7 \\
\hline S-FLDA & 98.3 & 100.0 & 100.0 & 99.2 & 99.7 \\
\hline
\end{tabular}

Table 4. Computational time of each method

\begin{tabular}{|c|c|c|c|c|}
\hline Dimension of data & 200 & 544 & 2500 & 5000 \\
\hline EVs & 3 & 3 & 4 & 4 \\
\hline No.of learning data & 40 & 40 & 30 & 30 \\
\hline Simple-FLDA[sec] & 0.06 & 0.21 & 1.19 & 2.375 \\
\hline Conventional FLDA[sec] & 0.1 & 0.84 & 21.6 & $\begin{array}{l}\text { Impossible } \\
\text { calculation }\end{array}$ \\
\hline
\end{tabular}

mulated contribution relevance is about $80[\%]$ when the number of eigenvectors is 10 for 10 persons and 15 for the others. Moreover, the number of trials is therefore 6.

Table 3 shows the result of the personal authentication experiment. In Table 3, "No.of subjects" means the number of subjects used for personal authentication experiment. As a result in Table 3, recognition accuracy by the Simple-FLDA is better than that by the Simple-PCA as well as the result (Table 2) of the first recognition experiment.

3.3 Computational Time In this section, the computational time of Simple-FLDA and the discriminant analysis based on matrix calculation of the past is compared. The computational time when the eigenvector is calculated is shown in Table 4 . In the conventional discriminant analysis, the eigenvectors are calculated by the power method.

When the dimension of data is small, the difference at the computational time is not seen in both the methods. However, it is understood that the computing time of Simple-FLDA is more effective as the dimension of data grows. Notice that, the computational time depends on the convergence condition of the repeated calculation.

\section{Conclusion}

In this paper, we proposed an approximation algorithm (Simple-FLDA) that sequentially calculated the eigenvectors by an easy iterative calculation without the use of the matrix calculation of the discriminant analysis. The eigenvectors to generate the feature can be sequentially calculated by using the Simple-FLDA based on a geometrical interpretation of the fisher linear discriminant analysis. This algorithm is easier than the algorithm based on the gradient method because our algorithm is derived in consideration of the meaning of the data distribution. Moreover, we carried out the computer simulations about recognition of wrist motion pattern by EMG measured from wrist and personal authentications that use face images to verify the effectiveness 
of this technique. As a result, recognition accuracy by the Simple-FLDA is better than that by the Simple-PCA in each experiment. It was confirmed that the SimpleFLDA was one of the effective techniques in the pattern recognition. We will confirm the effectiveness even for another problems in the future. Furthermore convergence property of the Simple-FLDA should be analyzed.

(Manuscript received Sep. 26, 2006)

\section{References}

(1) R.O. Duda and P.E. Hart: Pattern Classification and Scene Analysis, John Wiley \& Sons (1973)

( 2 ) B. Scholkopf, A. Smola, and K.R. Muller: "Nonlinear Component Analysis as a Kernel Eigenvalue Problem", Technical Report No.44, Max-Planck-Institute, Germany (1996)

( 3 ) M.H. Yang: "Kernel Eigenfaces vs. Kernel Fisherfaces : Face Recognition Using Kernel methods", Proc. of Fifth IEEE International Conference on Automatic Face and Gesture Recognition, pp.215-220, Washington, D.C. (2002)

(4) P.N. Belhumeur, J.P. Hespanha, and D.J. Kriegman: "Eigenfaces vs. Fisherfaces : Recognition Using Class Specific Linear Projection", IEEE Trans. on Pattern Analysis and Machine Intelligence, Vol.19, No.7, pp.711-720 (1997)

(5) T.D. Sanger: "Optimal Unsupervised Learning in a Single Layer Linear Feedforward Neural Network", Neural Networks, Vol.2, No.6, pp. 459-473 (1989)

(6) S.Y. Kung: Digital Neural Networks, Prentice-Hall (1993)

(7) M. Partridge and R. Calvo: "Fast dimentionality reduction and simple PCA", IDA, 2, pp.292-298 (1997)

(8) H. Takimoto, Y. Mitsukura, M. Fukumi, and N. Akamatsu: "A Feature Extraction Method for Personal Identification System by Using Real-Coded Genetic Algorithm", Proc. of 7th SCI' 2003, Orlando, USA, Vol.4, pp.66-77 (2003)

(9) M. Nakano, F. Yasukata, and M. Fukumi: "Recognition of Smiling Faces Using Neural Networks and SPCA", International Journal of Computational Intelligence and Applications, Vol.4, No.2, pp.153-164 (2004)

(10) S. Kuroiwa, S. Tsuge, H. Tani, X-Y. Tai, M. Shishibori, and K. Kita: "Dimensionality reduction of vector space model based on Simple PCA", Proc. Knowledge-Based Intelligent Information Engineering Systems \& Allied Technologies (KES), Vol.2, pp.362-366, Osaka, Sep. (2001)

(11) Y. Ishioka: Standard and Application of Stomatognathic Function Analysis, dental Diamond Company, pp.260-273 (1991) (in Japanese)

(12) Y. Matsumura, M. Fukumi, N. Akamatsu, and K. Nakaura: "Recognition of Wrist EMG Signal Patterns Using Neural Networks", Journal of Intelligent and Fuzzy Systems, 15, No.3-4, pp.165-171 (2004)

(13) M. Fukumi and Y. Mitsukura: "Feature Generation by Simple FLD", Proc. of 9th International Conference on Knowledge-Based Intelligent Information \& Engineering Systems, Sept.2005, Melboume, Australia (2005)

(14) M. Fukumi and Y. Mitsukura: "A Simple Feature Generation Method Based on Fisher Linear Discriminant Analysis", Proc. of IASTED International Conference on Signal and Information Processing 2005, pp.342-346, Honolulu, Hawaii (2005)
Tadahiro Oyama (Non-member) received B.E. degrees

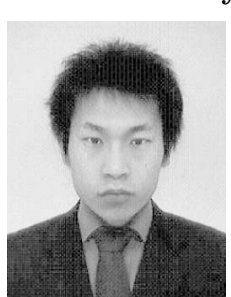
from the National Institution for Academic Degrees and University Evaluation, in 2005. He is currently master's course student with the Department of Information Science and Intelligent Systems, University of Tokushima. His research interests include pattern recognition and human sensing. He is a student member of the IEICE.

Yuji Matsumura (Non-member) received B.E. and M.E.

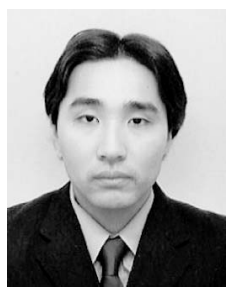
degrees from the University of Tokushima, in 2002 and 2004, respectively. He is currently pursuing the $\mathrm{Ph}$.D. degree at University of Tokushima. His research interests include biosignal processing. He is a student member of the Institute of Systems, Control and Information Engineers.

Stephen Githinji Karungaru (Non-member) received the

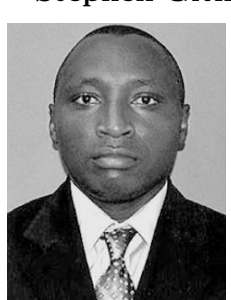
B.Sc. degree in electronics and communication from Moi University in 1992. He then joined the Department of Electrical and Electronics Engineering, Jomo Kenyatta University of Agriculture and Technology as a teaching assistant. He received the M.E. degree and a $\mathrm{PhD}$ in information system design from the Department of information science and Intelligent Systems, University of Tokushima in 2001 and 2004 respectively. He is currently an assistant professor in the same department. His research interests include pattern recognition, neural networks, evolutionally computation, image and signal processing and robotics. He is a member of IEEE and ACM.

Minoru Fukumi (Member) received the B.E. and M.E. de-

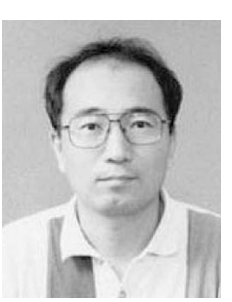
grees from the University of Tokushima, in 1984 and 1987, respectively, and the doctor degree from Kyoto University in 1996. Since 1987, he has been with the Department of Information Science and Intelligent Systems, University of Tokushima. In 2005, he became a Professor in the same department. He received the best paper award from the SICE in 1995 and best paper awards from some international conferences. His research interests include neural networks, evolutionary algorithms, image processing and human sensing. He is a member of the SICE, ISCIE, IPSJ and IEICE. 